\title{
Evaluation of Fluorotype MTB for detection of Mycobacterium tuberculosis complex DNA in clinical specimens from a low-incidence country
}

\author{
Sabine Hofmann-Thiel ${ }^{*}$ and Harald Hoffmann
}

\begin{abstract}
Background: With Fluorotype MTB (FT MTB, HAIN Lifesciences, Germany) a new semi-automated assay for detection of $M$. tuberculosis complex (MTBC) in clinical specimens has been introduced. In a prospective study, we evaluated the diagnostic performance of FT MTB in a routine diagnostic setting in a low-incidence country.

Methods: A total of 1039 respiratory specimens received for routine mycobacteriology diagnostics were analysed by FT MTB. Results were compared to those of culture, microscopy and clinical diagnosis. 61 specimens were excluded from further analysis due to bacterial contamination of cultures.

Results: FT MTB detected 52 of 59 TB specimens (45 culture-positive with MTBC, 7 with clinical diagnosis of TB). With 902 of 912 non-TB specimens (884 culture-negative, 18 with growth of non-tuberculous mycobacteria) FT MTB was negative; discrepant positive FT MTB results were found with 10 specimens. Overall sensitivity, specificity, positive and negative predictive values were $88.1 \%, 98.9 \%, 83.8 \%$ and $99.2 \%$. Sensitivity rates for smear-positive and smear-negative TB specimens were $100 \%$ and $56.3 \%$, respectively. Seven of 978 samples (0.7\%) yielded invalid FT MTB results.

Conclusions: FT MTB is a new accurate, half automated assay for rapidly diagnosing TB and suitable for larger series of samples. Performance characteristics were found to be similar to those of other commercial NAATs. Its sensitivity in paucibacillary, smear-negative specimens and its utility for TB diagnostics in high-incidence settings needs to be addressed in further studies.
\end{abstract}

Keywords: Mycobacterium tuberculosis complex, TB diagnostics, MTBC, NAAT, PCR, Nucleic acid amplification, Fluorotype MTB, Respiratory

\section{Background}

Since the introduction of rapid, easy to use nucleic acid amplification tests (NAATs), tuberculosis (TB) diagnostics is shifting world wide towards molecular tests. Among commercial assays for the detection of Mycobacterium tuberculosis complex (MTBC), Xpert MTB/RIF (Cepheid, USA) is securing the pole position particularly in low- and middle income countries with high burden of multi-drug resistant TB (MDR-TB). Due to the fully integrated cartridge design, the simple and safe workflow, and the ability to simultaneously detect MTBC and rifampicin (RMP) resistance, Xpert MTB/RIF allows rapid TB diagnosis and drug resistance testing also at the peripheral health clinic

\footnotetext{
* Correspondence: sabine.hofmann-thiel@synlab.com

synlab MVZ Gauting, IML red, WHO Supranational Reference Laboratory of Tuberculosis, Robert-Koch-Allee 2, 82131 Gauting, Germany
}

level $[1,2]$. In fully industrialized countries however, these strengths do not prove similarly effective. First, TB incidence as well as RMP resistance rate is comparably low. In Germany, for example, TB incidence is still declining reaching 5.3\% in 2011; MDR-TB rate is at around 2\% [3]. Second, the price per shot is high compared to other commercial assays. Finally, TB NAATs are often processed in large batches in which Xpert MTB/RIF would not offer significant time savings. Therefore, semi-automated assays designed for larger series are more attractive for many mycobacteriology laboratories in low-prevalence settings. Widely used semi-automated assays are, for example, COBAS TaqMan MTB (CTM-MTB) (Roche, Germany), BD probeTEC ET (probeTEC, Becton-Dickenson, USA) or Gen-Probe Amplified MTB Direct Test (AMDT, Gene-Probe, USA). Most of them are able to detect 
MTBC with specificities of nearly $100 \%$ whereas sensitivities appear more variable, in particular with paucibacillar specimens [4-7]. In direct comparison studies, performance characteristics have been shown to be in the range of that of Xpert MTB/RIF [8-10]. While probeTEC and AMDT are demanding many open work steps with certain risk of cross-contamination, CTM-MTB is, so far, the only one considered to be semi-automated using a real-time PCR platform [5].

Most recently, a new semi-automated assay has been introduced for rapid detection of MTB complex from respiratory and non-respiratory clinical specimens. The FluoroType MTB (FT MTB, HAIN Lifescience, Germany) is a HyBeacon-based PCR assay performed on a Fluorocycler instrument allowing amplification and detection in a closed system. The aim of our present study was to comprehensively evaluate FT MTB for the routine detection of MTBC in respiratory specimens and to compare the results to conventional culture, microscopy and clinical data.

\section{Methods \\ Clinical specimens}

In total, 1039 respiratory specimens (390 sputa, 556 bronchial aspirates, 89 bronchoalveaolare lavages, 4 tracheal secretes) were included in the study. They originated from patients with suspected infection by MTBC or nontuberculous mycobacteria (NTM) and were sent for routine mycobacteriology diagnostics between March and July 2012. The study protocol, involving the use of clinical specimens and human data, has been approved by the ethics committees of the Bayrische Landesärztekammer (no. 06043) and the Ludwig-Maximilian University of Munich (no. 437-12). In the scope of an open prospective study, consecutive specimens were taken provided that (i) sputum material was of sufficient quality, (ii) enough material was available to perform Fluorotype MTB assay in addition to requested routine analyses, (iii) material from the same patient has not been tested more than one time before and (iv) the material did not come from a known TB patient under therapy or with a known history of $\mathrm{TB}$, provided this information was available at the time of enrolment.

All specimens were decontaminated using $\mathrm{N}$-acetyl-Lcysteine (NALC)-NaOH. After concentration by centrifugation, the sediment was resuspended in $1.5 \mathrm{ml}$ of $0.5 \mathrm{M}$ phosphate buffer ( $\mathrm{pH}$ 6.8) and inoculated in liquid (MGIT ${ }^{\mathrm{m}}$, Becton-Dickinson, Heidelberg, Germany), Loewenstein-Jensen and Stonebrinck medium. Smears were prepared from this suspension, stained with Auramin $\mathrm{O}$ and visualized with a fluorescence microscope (magnification $\times 400$ ). From the leftover suspension DNA was prepared and used directly for Fluorotype MTB assay or stored frozen.
Cultures were incubated for up to 8 weeks. In case a primary culture turned positive with acid fast bacilli, the isolate was identified by DNA line probe assays (Genotype CM, Genotype MTBC, Hain Lifesciences, Nehren, Germany) or by sequencing of the $16 \mathrm{~S}$ rRNA gene.

\section{DNA preparation}

Fully automated isolation of DNA was done using the GenoXtract automat (HAIN Lifesciences) and the GTX DNA/RNA Extraction Kit, according to the instructions of the manufacturer. In brief, $700 \mu \mathrm{l}$ decontaminated material was transferred into a screw cap container and heat inactivated at $100^{\circ} \mathrm{C}$ for $20 \mathrm{~min}$. Subsequently, pump-pipette-tip units, reagent cartridges, and elution containers were mounted in the GenoXtract machine. The protocol was started. After about $40 \mathrm{~min}$ the ready-to-use DNA was isolated in a volume of $100 \mu$ l elution buffer.

\section{Fluorotype MTB assay}

Fluorotype MTB test is performed on a FluoroCycler (HAIN Lifesciences) and combines DNA amplification using specific primers and subsequent melting curve analysis in one instrument. First, PCR mixes were freshly prepared by combining $3 \mu \mathrm{l}$ amplification mix A (AM-A) with $7 \mu$ amplification mix $B(A M-B)$ in the preamplification room I. Subsequently, $6 \mu \mathrm{l}$ of isolated DNA was added in the pre-amplification room II. Controls included $6 \mu \mathrm{l}$ of PCR-grade water (negative control) and $6 \mu \mathrm{l}$ of control DNA C + FT MTB (positive control). PCR mixes were immediately loaded into the FluoroCycler. Results were available after 2 hours $10 \mathrm{~min}$. The FluoroSoftware automatically analyzed melting curves for the amplification control $(\mathrm{AC})\left(70.5^{\circ} \mathrm{C}+/-3^{\circ} \mathrm{C}\right)$ and for MTBC (melting point $60.0^{\circ} \mathrm{C}+/-3^{\circ} \mathrm{C}$ ) and computed results as "no MTB complex DNA detected" (MTBC negative, AC positive), "MTB complex DNA detected" (MTBC positive, AC positive or negative), "not interpretable" in case of unspecific peaks, or "invalid" in case of failure of positive/negative controls or AC.

\section{Analysis of results}

FT MTB results were initially compared to outcomes of the cultures. In cases of discrepant results, clinical, histological and anamnesis data of the patients were included in the final evaluation. For example, an initially discrepant positive FT MTB result (FT MTB positive, culture negative) was re-classified "true positive" if the patient was finally diagnosed as TB case by other means, i.e. by combinations of medical history, clinical examination, radiology, histopathology, and treatment success with anti-TB regimens. Invalid or not interpretable results were excluded from the final calculation of sensitivity, specificity and prospective values. Calculations of positive and negative predictive values were based on 
the detected prevalence of 6\% (59/971), which is largely in agreement with prevalence values found in laboratories of many low-prevalence countries. 95\% confidence intervals were determined using the Vassar-Stats calculator. Statistical analyses were performed using unpaired $t$ tests on the OpenEpi version 2.3 platform. A $P$ value of $<0.05$ was considered statistically significant.

\section{Results}

\section{Comparison of FT MTB with culture data}

In total, 1039 respiratory specimens were analysed by FT MTB and, in parallel, subjected to microscopy and culture. 908 of 1039 specimens (87.4\%) were culturenegative, 18 (1.7\%) yielded growth of NTMs $(9 M$. intracellulare, $4 M$. gordonae, $2 M$. avium, $2 M$. fortuitum, 1 M. abscessus), and 52 (5.0\%) growth of MTBC (51 MTB, 1 M. caprae) (Table 1). Of the latter ones, 42 specimens (86.7\%) were smear-positive. Bacterial contamination of liquid or solid cultures was observed in 61 of 1039 (5.8\%) cases. They were excluded from further analysis.

Of the remaining 978 specimens, FT MTB results were negative in 909 (92.9\%) and positive in $62(6.3 \%)$ cases (Table 1). Seven (0.7\%) specimens yielded invalid FT MTB results. 902 of 909 (99.2\%) negative FT MTB results matched with culture: 884 specimens yielded negative culture and from 18 specimens NTM grew (Table 1). Discrepant results were found with seven FT MTB negative specimens, which were all smear-negative but MTB grew in liquid culture; they were considered false-negative. 45 of $62(72.6 \%)$ FT MTB positive specimens were in agreement with culture results: they grew MTBC in culture and $93.3 \%$ of them $(42 / 45)$ were smear-positive. 17 of 62 (27.4\%) FT MTB positive specimens were culture-negative and thus considered "discrepantly positive". All but one of them $(94.1 \%)$ showed a smear negative microscopy.

\section{Analysis of discrepant results}

All 17 discrepantly positive FT MTB derived from patients without positive culture from any materials sent to our laboratory, i.e. none of the patients were

Table 1 Comparison of FT MTB and culture results

\begin{tabular}{lcccc}
\hline & \multicolumn{4}{c}{ No. of specimens with FT MTB results } \\
\cline { 2 - 5 } Culture results $^{\text {a }}$ & Pos & Neg & Invalid & Total \\
\hline MTBC & 45 & 7 & 0 & 52 \\
$\quad$ Smear positive & 42 & 0 & 0 & 42 \\
$\quad$ Smear negative & 3 & 7 & 0 & 10 \\
No MTBC & 17 & 902 & 7 & 926 \\
$\quad$ Culture negative & 17 & 884 & 7 & 908 \\
$\quad$ Growth of NTM & 0 & 18 & 0 & 18 \\
Total & 62 & 909 & 7 & 978 \\
\hline
\end{tabular}

${ }^{\mathrm{a} M T B C}$, Mycobacterium tuberculosis complex; NTM, non-tuberculous mycobacteria; pos, positive; neg, negative. registered in our records as a TB case. Nonetheless, seven cases were diagnosed with $\mathrm{TB}$ after review of the patients' charts. Consequently, we re-classified the results from 7 of 17 specimens as "true-positive". Two of them were recovered from patients with clinically and histological confirmed tuberculosis although cultures consistently remained negative. Further two specimens were recovered from patients already receiving TB therapy at the time of specimen collection; one of them was smearpositive. The patients' records indicated that other mycobacteriology laboratories reported positive cultures from previous specimens. Three specimens were obtained from patients with a recent history of TB. All three had already successfully completed TB therapy within the previous 10 months and requested now diagnostic work-up for the suspicion of relapse. The remaining 10 specimens with discrepantly positive FT MTB originated from patients without any documented history or diagnosis of $\mathrm{TB}$ and were finally classified false-positive (Table 2).

Although FT MTB is a qualitative assay, signal values of melting curve peaks can be read from the corresponding graphs. Notably, false-positive results $(n=10)$ showed significantly lower MTBC-specific peak values (mean 257 units; standard deviation [SD] \pm 121.6 units) compared to that of true-positive results (mean 650 units; $\mathrm{SD} \pm 87.5)(p<0.001)$ (Figure 1). All but one of falsepositive results showed values below 320 suggesting the existence of a critical zone of "low grade positivity".

\section{Sensitivity and specificity}

After resolving discrepant and excluding invalid values, 10 out of 912 "no TB" specimens yielded positive FT MTB results corresponding to a specificity of $98.9 \%$ (Table 2). 52 out of 59 "TB" specimens yielded positive FT MTB results corresponding to an overall sensitivity of $88.1 \%$. With smear-negative specimens, sensitivity was $56.3 \%$ (9/16), with smear positive ones 100\% (43/43) (Table 2). Based on a prevalence of $6 \%$ TB specimens among the 971 study samples, positive and negative predictive values were calculated to $83.8 \%$ and $99.2 \%$, respectively.

\section{Discussion}

The FT MTB assay is a new semi-automated assay for detection of MTBC giving results within 3-4 hours. Similar to other semi-automated systems like COBAS TaqMan MTB, different workstations are required including DNA extraction, preparation of PCR mixes and amplification/detection. DNA extraction can be done automated using the GenoXtract instrument requiring only minimal hands-on time. Furthermore, amplification and detection is performed fully-automated in the Fluorocycler instrument; the handling of the instrument is simple and the analysis software designed userfriendly. Including all work steps, hands-on time for 12 
Table 2 Resolved results for sensitivity, specificity, PPV and NPV of FT MTB (culture and clinical diagnosis)

\begin{tabular}{|c|c|c|c|c|c|c|c|c|}
\hline \multirow[b]{3}{*}{ Smear microscopy } & \multicolumn{4}{|c|}{$\begin{array}{l}\text { No. of specimens with indicated results by } \\
\text { culture and clinical data }(n=971)^{\mathrm{a}}\end{array}$} & \multirow[b]{3}{*}{$\begin{array}{c}\text { Sensitivity } \\
(95 \% \mathrm{Cl})\end{array}$} & \multirow[b]{3}{*}{$\begin{array}{l}\text { Specificity } \\
(95 \% \mathrm{Cl})\end{array}$} & \multirow[b]{3}{*}{$\begin{array}{c}\text { PPV } \\
(95 \% \mathrm{Cl})\end{array}$} & \multirow[b]{3}{*}{$\begin{array}{c}\text { NPV } \\
(95 \% \mathrm{Cl})\end{array}$} \\
\hline & \multicolumn{2}{|c|}{ TB $(n=59)$} & \multicolumn{2}{|c|}{ no TB $(n=912)$} & & & & \\
\hline & $\begin{array}{c}\text { FT MTB pos } \\
(\%)\end{array}$ & $\begin{array}{c}\text { FT MTB neg } \\
\text { (\%) }\end{array}$ & $\begin{array}{c}\text { FT MTB pos } \\
(\%)\end{array}$ & $\begin{array}{c}\text { FT MTB neg } \\
\text { (\%) }\end{array}$ & & & & \\
\hline \multirow[t]{2}{*}{ Smear positive } & 43 & 0 & 0 & 3 & 100 & 100 & 100 & 100 \\
\hline & $(82.7)$ & (0) & (0) & $(0.3)$ & $(89.7-100)$ & $(30.9-100)$ & $(89.7-100)$ & $(30.9-100)$ \\
\hline \multirow[t]{2}{*}{ Smear negative } & 9 & 7 & 10 & 899 & 56.3 & 98.9 & 47.3 & 99.2 \\
\hline & $(17.3)$ & $(100)$ & $(100)$ & $(99.7)$ & $(30.5-79.2)$ & $(97.9-99.4)$ & $(25.2-70.5)$ & (98.3-99.6) \\
\hline \multirow[t]{2}{*}{ All } & 52 & 7 & 10 & 902 & 88.1 & 98.9 & 83.8 & 99.2 \\
\hline & & & & & $(76.4-94.6)$ & $(97.9-99.4)$ & $(72.8-91.5)$ & $(98.3-99.6)$ \\
\hline
\end{tabular}

${ }^{\mathrm{a}} \mathrm{TB}$, tuberculosis; pos, positive; neg, negative; PPV, positive predictive value; NPV, negative predictive value.

specimens adds up to approximately $30 \mathrm{~min}$ being comparable to other semi-automated systems [10]. The Fluorocycler system is suitable for low numbers of samples as well as for large series, as up to 8 Fluorocycler instruments (each with 12 positions) can be operated simultaneously by one control computer. In comparison, the Xpert MTB/RIF system enables hands-on time of less than 3 min per specimens being of advantage only when handling a small number of samples.

The aim of the present study was to assess the performance characteristics of the FT MTB assay in a routine setting of a German mycobacteriology laboratory. FT MTB results from 978 respiratory specimens were compared to culture and clinical diagnosis as "gold standard". The specificity (98.9\%) of FT MTB was in the range of that of other NAATs $(98.4 \%-99.7 \%$, mean 99.1\%) [2,4-6,11,12].

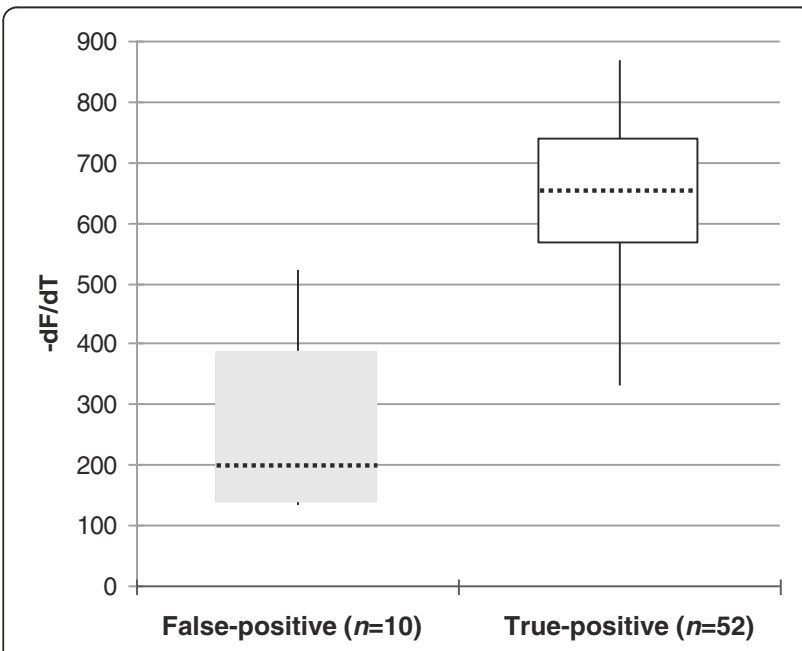

Figure 1 Comparison of false-positive and true-positive results. MTBC-specific melting curve peak values for false-positive (left, $n=10$ ) and true-positive (right, $n=52$ ) FT MTB results are shown. Boxes in grey (false-positive) and white (true-positive) represent means of values \pm standard deviation, respectively. Maximum and minimum values are depicted by vertical lines. Dotted lines show median values.
In particular, no cross-reactivity with 18 specimens growing NTM was observed. Ten positive FT MTB results remained unresolved, even after review of patients `clinical data. It remains elusive whether these false-positive outcomes are caused by residual MTBC DNA despite absence of active disease, as speculated before [13], or by unspecific amplification. Although reported as positive by the manufacturers' analysis software, false-positive FT MTB tests seem to give lower MTBC-specific melting curve peaks than true-positive ones. Discrepant positive results in the "zone of low-positivity" are reported also for other NAAT systems [14-16]. A repeat testing is, therefore, generally recommended for specimens with low positivity values before transferring a final laboratory report to the clinical doctor.

The overall sensitivity of FT MTB reached $88.1 \%$ being in the upper range $(63.2-95.0 \%$, mean $84.2 \%)$ of levels reported for other commercial NAATs like BD ProbeTec ET (63.2-86.2\%) [6,11], COBAS Taqman MTB (81.1-91.5\%) [4,5], GeneProbe AMTD (95\%) [12], Speed-oligo direct MTB (76\%) [17] or Xpert MTB/RIF (88.0-92.2\%) [2,18].

Considering smear-positive TB specimens only, sensitivity and PPV reached 100\%. In comparison, sensitivity levels reported for other commercial assays were 95.5\% to $100 \%$ [2,5,6,17-19]. A high positive predictive value for smear-positive specimens is particularly important in settings where NTM are common. Particularly in fully industrialized countries, NTM are increasingly isolated from clinical specimens and reported as cause of opportunistic infections mainly in immunocompromised individuals [20-22]. Therefore, modern NAAT assays should rapidly and reliably differentiate between TB and NTM infection, particularly in patients with immunodeficiency or in patients with first smear-positive sputa and unspecific clinical signs of TB. The FT MTB obviously fulfils this requirement.

Considering smear-negative TB specimens only, the sensitivity of FT MTB was $56.3 \%$. Of the smear-negative TB samples which were missed by FT MTB, four 
exhibited positive culture results only after $\geq 24$ days of incubation, indicating a paucibacillary nature of the specimens. This doesn't mitigate the fact, that we found a sensitivity level for smear-negative samples which was in the lower range of values reported for other NAATs (49.1-86.7\%, mean 64.1\%) $[2,4,6,12,17,18]$. However, the low absolute number of smear-negative TB samples $(n=16)$ included in the present study might have biased the outcomes for such samples. Consequently, the sensitivity of FT MTB for paucibacillary specimens should be re-assessed in further, preferably multi-centre studies with higher numbers of smear-negative TB samples.

\section{Conclusions}

In summary, FT MTB has proven to be a fast, easy and accurate new assay for direct detection of MTBC. The assay yielded good performance characteristic in terms of overall sensitivity and specificity with a potential weakness regarding sensitivity in smear-negative specimens which needs to be addressed in further investigations. Due to its user-friendly design, the closed system for amplification and detection and the possibility of simultaneously processing up to 96 samples without significant loss of time, FT MTB is an alternative to other semi- or fully automated assays particularly in low-prevalence countries.

\section{Competing interests}

The authors declare that they have no competing interests.

\section{Authors' contributions}

SH-T supervised the study and laboratory work, performed data analysis and drafted the manuscript. H.H. participated in study design and participated in preparation of the manuscript. Both authors read and approved the final manuscript.

\section{Acknowledgements}

The study was supported by a grant from Kuratorium Tuberkulose in der Welt e.V., München-Gauting, Germany. We thank Beate Witzler for excellent technical assistance. We are also grateful to HAIN Lifesciences for providing instrumentation and test kits (FT MTB \& GenoXtract) free of charge.

Received: 5 October 2013 Accepted: 30 January 2014

Published: 5 February 2014

\section{References}

1. Helb D, Jones M, Story E, Boehme C, Wallace E, Ho K, Kop J, Owens MR, Rodgers R, Banada P, Safi H, Blakemore R, Lan NT, Jones-López EC, Levi M, Burday M, Ayakaka I, Mugerwa RD, McMillan B, Winn-Deen E, Christel L, Dailey P, Perkins MD, Persing DH, Alland D: Rapid detection of Mycobacterium tuberculosis and rifampin resistance by use of on-demand, near-patient technology. J Clin Microbiol 2010, 48:229-237.

2. Boehme CC, Nabeta P, Hillemann D, Nicol MP, Shenai S, Krapp F, Allen J, Tahirli R, Blakemore R, Rustomjee R, Milovic A, Jones M, O'Brien SM, Persing DH, Ruesch-Gerdes S, Gotuzzo E, Rodriques C, Alland D, Perkins MD: Rapid molecular detection of tuberculosis and rifampin resistance. N Engl J Med 2010, 363:1005-1015.

3. Robert Koch Institut: Bericht zur Epidemiologie der Tuberkulose in Deutschland für 2011. Berlin: Robert Koch Institut: 2013.

4. Tortoli E, Urbano P, Marcelli F, Simonetti TM, Cirillo DM: Is real-time PCR better than conventional PCR for Mycobacterium tuberculosis complex detection in clinical samples?. J Clin Microbiol 2012, 50:2810-2813.
5. Yang YC, Lu PL, Huang SC, Jenh YS, Jou R, Chang TC: Evaluation of the Cobas TaqMan MTB test for direct detection of Mycobacterium tuberculosis complex in respiratory specimens. J Clin Microbiol 2011, 49:797-801.

6. Goessens WHF, de Man P, Koeleman GM, Luijendijk A, te Witt R, Endtz HP, van Belkum A: Comparison of the COBAS AMOLICOR MTB and BDProbeTec ET assays for detection of mycobacterium tuberculosis in respiratory specimens. J Clin Microbiol 2005, 43:2563-2566.

7. Piersimoni C, Callegaro A, Scarparo C, Penati V, Nista D, Bornigia S, Lacchini C, Scagnelli M, Santini G, De Sio G: Comparative evaluation of the new gen-probe Mycobacterium tuberculosis amplified direct test and the semiautomated abbott LCX Mycobacterium tuberculosis assay for direct detection of Mycobacterium tuberculosis complex in respiratory and extrapulmonary specimens. J Clin Microbiol 1998, 6:3601-3604.

8. Causse M, Ruiz P, Gutierrez-Aroca JB, Casal M: Comparison of two molecular methods for rapid diagnosis of extrapulmonary tuberculosis. $J$ Clin Microbiol 2011, 49:3065-3067.

9. Park KS, Kim JY, Lee JW, Hwang YY, Jeon K, Koh WJ, Lo CS, Lee NY: Comparison of the Xpert MTB/RIF and Cobas TaqMan MTB Assays for Detection of Mycobacterium tuberculosis in Respiratory Specimens. J Clin Microbiol 2013, 51:3225-3227.

10. Antonenka U, Hofmann-Thiel S, Turaev L, Esenalieva A, Abdulloeva M, Sahalchyk E, Alnour T, Hoffmann H: Comparison of Xpert MTB/RIF with ProbeTec ET DTB and COBAS TaqMan MTB for direct detection of M. tuberculosis complex in respiratory specimens. BMC Infect Dis 2013, 13:280.

11. Luh KT, Hsueh PR, Hsu HL, Lee LN, Wang JY: Performance assessment of the DR.MTBC Screen assay and the BD ProbeTec ET system for direct detection of Mycobacterium tuberculosis in respiratory specimens. J Clin Microbiol 2006, 44:716-719.

12. Piersimoni C, Callegaro A, Nista D, Bornigia S, De Conti F, Santini G, De Sio G: Comparative evaluation of two commercial amplification assays for direct detection of Mycobacterium tuberculosis complex in respiratory specimens. J Clin Microbiol 1997, 35:193-196.

13. Reischl U, Lehn N, Wolf H, Naumann L: Clinical evalution of automated COBAS AMPLICOR MTB assay for testing respiratory and nonrespiratory specimens. J Clin Microbiol 1998, 36:2853-2860.

14. Rüsch-Gerdes S, Richter E: Clinical evaluation of the semiautomated BDProbeTec ET System for the detection of Mycobacterium tuberculosis in respiratory and nonrespiratory specimens. Diagn Microbiol Infect Dis 2004, 48:265-270.

15. Kim JH, Kim YJ, Ki CS, Kim JY, Lee NY: Evaluation of Cobas TaqMan MTB PCR for detection of Mycobacterium tuberculosis. J Clin Microbiol 2011, 49:173-176.

16. Kerleguer A, Koeck JL, Fabre M, Gerome P, Teyssou R, Herve V: Use of equivocal zone in interpretation of results of the amplified mycobacterium tuberculosis direct test for diagnosis of tuberculosis. J Clin Microbiol 2003, 41:1783-1784

17. Lara-Oya A, Mendoza-Lopez P, Rodriquez-Granger J, Fernandez-Sanchez AM, Bermudez-Ruiz MP, Toro-Peinado I, Palop-Borrás B, Navarro-Marí JM, Martínez-Lirola MJ: Evaluation of the speed-oligo direct Mycobacterium tuberculosis assay for molecular detection of mycobacteria in clinical respiratory specimens. J Clin Microbiol 2013, 51:77-82.

18. Rachow A, Zumla A, Heinrich N, Rojas-Ponce G, Mtafya B, Reither K, Ntinginya EN, O'Grady J, Huggett J, Dheda K, Boehme C, Perkins M, Saathoff E, Hoelscher M: Rapid and accurate detection of Mycobacterium tuberculosis in sputum samples by Cepheid Xpert MTB/RIF assay-a clinical validation study. PLOS ONE 2011, 6:e20458.

19. Lebrun L, Mathieu D, Saulnier C, Nordmann P: Limits of commercial molecular tests for diagnosis of pulmonary tuberculosis. Eur Respir J 1997, 10:1874-1876.

20. Moore JE, Kruijshaar ME, Ormerod LP, Drobniewski F, Abubakar I: Increasing reports of non-tuberculous mycobacteria in England, Wales and Northern Ireland, 1995-2006. BMC Public Health 2010, 10:612.

21. Thomson RM: Changing epidemiology of pulmonary nontuberculous mycobacteria infections. Emerg Infect Dis 2010, 16:1576-1583.

22. Cassidy PM, Hedberg K, Saulson A, McNelly E, Winthrop KL: Nontuberculous mycobacterial disease prevalence and risk factors: a changing epidemiology. Clin Infect Dis 2009, 49:124-129.

doi:10.1186/1471-2334-14-59

Cite this article as: Hofmann-Thiel and Hoffmann: Evaluation of Fluorotype MTB for detection of Mycobacterium tuberculosis complex DNA in clinical specimens from a low-incidence country. BMC Infectious Diseases 2014 14:59. 